\title{
Diabetic-induced alterations in hepatic glucose and lipid metabolism: The role of type 1 and type 2 diabetes mellitus (Review)
}

\author{
SAIZHI JIANG ${ }^{1,2}$, JAMIE L. YOUNG ${ }^{3}, \mathrm{KAI} \mathrm{WANG}^{1,2}$, YAN QIAN $^{1}$ and $\mathrm{LU} \mathrm{CAI}^{2-4}$ \\ ${ }^{1}$ Department of Paediatrics, The First Affiliated Hospital of Wenzhou Medical University, Wenzhou, \\ Zhejiang 325000, P.R. China; ${ }^{2}$ Department of Paediatrics, Paediatric Research Institute; \\ ${ }^{3}$ Department of Pharmacology and Toxicology; ${ }^{4}$ Radiation Oncology, University of Louisville, Louisville, KY 40202, USA
}

Received July 12, 2019; Accepted March 6, 2020

DOI: $10.3892 / \mathrm{mmr} .2020 .11175$

\begin{abstract}
Diabetes mellitus (DM) is a growing health concern in society. Type 1 and type $2 \mathrm{DM}$ are the two main types of diabetes; both types are chronic diseases that affect glucose metabolism in the body and the impaired regulation of glucose and lipid metabolism promotes the development and progression of DM. During the physiological metabolism process, the liver serves a unique role in glucose and lipid metabolism. The present article aimed to review the association between DM and glucose metabolism in the liver and discuss the changes of the following hepatic glucose fluxes: Gluconeogenesis, glucose/glucose 6-phosphate cycling, glycogenolysis, glycogenesis and the pentose phosphate pathway. Moreover, the incidence of fatty liver in DM was also investigated.
\end{abstract}

Correspondence to: Dr Saizhi Jiang, Department of Paediatrics, The First Affiliated Hospital of Wenzhou Medical University, Nanbaixiang, Ouhai, Wenzhou, Zhejiang 325000, P.R. China E-mail: alice.jiang@foxmail.com

Abbreviations: T2DM, type 2 diabetes mellitus; T1DM, type 1 diabetes mellitus; NAFLD, non-alcoholic fatty liver disease; TG, triglycerides; G/G6P, glucose/glucose 6-phosphate; GK, glucokinase; G6Pase, glucose 6-phosphatase; STZ, streptozotocin; 13C-NMRS, 13C nuclear magnetic resonance spectroscopy; HbAlc; glycated hemoglobin; GP, glycogen phosphorylase; GS, glycogen synthase; HGP, hepatic glucose production; TCA cycle, tricarboxylic acid cycle; PPP, pentose phosphate pathway; G6PD, glucose-6-phosphate dehydrogenase; L-PK, liver pyruvate kinase; FAS, fatty acid synthase; ACC, acetyl-CoA carboxylase; PFK1, phosphofructokinase; GAPDH, Glyceraldehyde 3-phosphate dehydrogenase; NADH, nicotinamide adenine dinucleotide; NADPH, nicotinamide adenine dinucleotide phosphate; $\mathrm{NADP}^{+}$, nicotine adenine dinucleotide phosphate

Key words: diabetes, sugar, insulin resistance, glucose metabolism, lipid metabolism

\section{Contents}

1. Introduction

2. Metabolic processing of glucose in the liver under diabetic conditions

3. Conclusion

\section{Introduction}

Glucose plays an important role in metabolism. It is not only the source of energy, but also the substrate of cell composition biosynthesis (1). The metabolic dysregulation of glucose homeostasis is the main consequence of the development of diabetes and the major cause of diabetic morbidity and mortality. There are two common types of diabetes: Type 1 and type 2. Type 1 diabetes mellitus (T1DM) is characterized by the insufficient secretion of insulin and the excessive release of glucagon, which promotes hepatic lipolysis and ketogenesis (2) and counteracts hepatic anabolism. Type 2 diabetes mellitus (T2DM) is the more common type of DM and is characterized by undetectable levels of insulin, increased liver fat content, impaired insulin clearance and hepatic insulin resistance (3).

DM is associated with various liver abnormalities, including non-alcoholic fatty liver disease (NAFLD) (3) and excessive hepatic glycogenosis (4). The liver serves a unique role in glucose metabolism and is crucial for systemic glucose homeostasis (5); it contributes to the management of an enteral glucose load by inhibiting its own glucose output, which aids the disposal of exogenous glucose by extrahepatic tissues, such as adipose and skeletal muscle (6). The dysregulation of liver signaling and metabolism predisposes individuals to NAFLD and/or T2DM, thus certain liver-derived biomarkers (fetuin-A, alpha-hydroxybutyrate and C-reactive protein) can be used for the diagnosis and prognosis of DM and DM-associated complications (7). Therefore, the liver is also an important target organ that regulates glucose homeostasis and can be targeted by the administration of specific diabetic drugs (8).

In the present review, the physiology and molecular pathways of liver glucose homeostasis were investigated, as well as 
the glucose metabolic disorders noted in DM. Hepatic lipogenesis was also investigated since lipid-induced hepatic insulin resistance is one of the main pathophysiological processes of hepatic glucose metabolism disorder in T2DM.

\section{Metabolic processing of glucose in the liver under diabetic conditions}

During glucose homeostasis, the liver serves an important role in carbohydrate synthesis, storage and redistribution (9). The liver performs opposite functions during hyperglycemic (glucose uptake and glycogen synthesis) and hypoglycemic states (glycogenolysis and gluconeogenesis), thus the physiological regulation of hepatic glucose production is a complex process (6). Patients with T2DM and T1DM demonstrate increased hepatic glucose production (HGP), of which multiple extrahepatic mechanisms contribute to the physiological regulation of HGP (10). However, DM is a bi-hormonal disease and is not simply the result of insulin deficiency $(11,12)$. The pancreatic endocrine cell hormones, glucagon (13) and insulin (14), both serve central roles in the regulation of both glucose and lipid metabolism. Insulin inhibits the secretion of glucagon and promotes the storage of lipids and carbohydrates, whereas glucagon facilitates gluconeogenesis and glucose efflux from the liver (15). Previous studies have reported that every type of diabetes is associated with hyperglucagonemia, the suppression of which can reduce hyperglycemia $(10,16)$. For example, insulin exerts a strong regulatory effect on the secretion of glucagon and causes the suppression of secretion of glucagon from pancreatic $\alpha$-cells; it has been reported that the absence of this paracrine regulatory mode contributes to the development of hyperglucagonemia and the increase of HGP in DM $(11,17)$. The main processes that contribute to glucose homeostasis, include glycogenolysis, glycogen synthesis, glycolysis and gluconeogenesis, all of which are regulated by independent mechanisms $(6,9)$. The dysregulation of these processes is discussed in detail in the following section.

Increased hepatic glucose/glucose 6-phosphate (G/G6P) cycling in DM. In hepatocytes, glucose is converted into G6P by glucokinase (GK; the gatekeeper for glucose metabolism in hepatocytes), and G6P is subsequently trapped in hepatocytes (8). The affinity of GK (also known as hexokinase IV) for glucose is low (S0.5; half-saturating concentration, $\sim 5 \mathrm{mmol} / \mathrm{l}$ ) and the reaction rate exhibits a sigmoidal dependence on the intracellular glucose concentration (18). When the blood glucose levels are $<5 \mathrm{mmol} / \mathrm{l}(90 \mathrm{mg} / \mathrm{dl})$, GK will not stimulate the production of large amounts of G6P and subsequent steps are blocked to ensure that hepatic glycogen synthesis is only highly active when blood glucose levels are high (19). G6P is subsequently converted to excess glycogen when insulin levels are high enough to activate glycogen synthase (GSb) and inactivate glycogen phosphorylase (GPa) (Fig. 1). In a diabetic state, it was demonstrated that the glucose uptake in hepatocytes from diabetic mice was significantly lower compared with the control animals, which may be a result of the repressed GK synthesis in response to a decreased insulin: Glucagon ratio (20). Glucagon has been demonstrated to modify hepatic glucose uptake; for example, a previous animal study indicated that under hyperglycemic and hyperinsulinemic conditions, the physiological changes noted in arterial blood glucagon dramatically changed the net hepatic glucose balance (21), whereas another study revealed that elevated blood glucagon levels impaired the ability of the liver to absorb and store glucose (22). Elevated blood-glucose in the postprandial state was found to activate GK activity and subsequently increase hepatic G6P production (23) (Fig. 1). In T2DM (24), impaired liver glucose uptake has been reported, which subsequently lead to postprandial hyperglycemia following the discovery that faulty hepatic GK activation could lead to impaired glucose uptake in T2DM (25). A more recent study demonstrated that the nicotinamide adenine dinucleotide (NAD)-dependent protein deacetylase sirtuin-2 (Sirt2) promoted hepatic glucose uptake through deacetylating the GK regulatory protein. Similary, in high fat diet-fed obese diabetic mice, the overexpression of hepatic Sirt2 increased the glucose uptake in the liver, attenuating impaired glucose tolerance (26).

Elevated G6P levels promote the upregulation of glucose 6-phosphatase (G6Pase) (27) and the gene expression levels of lipogenic enzymes, such as liver pyruvate kinase (L-PK), fatty acid synthase (FAS), and acetyl-CoA carboxylase (ACC) (28). Elevated levels of G6Pase suppress G6P levels during the fasting state (29). In the fed state, G6P is the precursor of glycogen synthesis and it is also metabolized to generate pyruvate through glycolysis (9). The alterations in the G/G6Pcycle have been observed in both humans and animals with T2DM $(30,31)$ as well as in animals with T1DM (20). For example, a previous study compared the levels of glucose in isolated hepatocytes from fasted, normal rats with the corresponding levels in hepatocytes from streptozotocin (STZ)-induced diabetic rats. The results indicated that glucose was absorbed by the liver and phosphorylated in normal fasting rats, whereas in diabetic hepatocytes, additional levels of the G6P precursor were subsequently dephosphorylated and released into the blood circulation (32). Thus, through downregulating the expression levels of GK or upregulating the activity of G6Pase, the concentration of G6P is disturbed, which may be the reason for the decline noted in the glucose metabolism responsiveness of diabetic subjects $(20,23,33)$.

Overall, the imbalance between hepatic glucose release and glucose uptake is an important factor in the development of DM; therefore, the drugs used to treat of DM should be designed to increase glucose uptake by activation of GK. Unfortunately, the majority of the drugs that target GK activation for the treatment of T2DM have not been clinically successful due to side effects, such as hypoglycemia, steatohepatitis and loss of efficacy over time (8). However, TTP399, a hepato-elective GK activator, was assessed in a recently reported double-blind, 6-month study and it was found that TTP399 did not cause hypoglycemia and exhibited limited or no detrimental effect on plasma lipids or liver enzymes (34). Moreover, it is a liver specific GK activator without side effects of hypertension, highlighting the importance of liver selectivity when targeting GK activity (34).

Hepatic glycogenesis is reduced in T2DM, whilst in T1DM, it is associated with insulin therapy. Net glycogen deposition in the liver depends on the coordinated inhibition of 


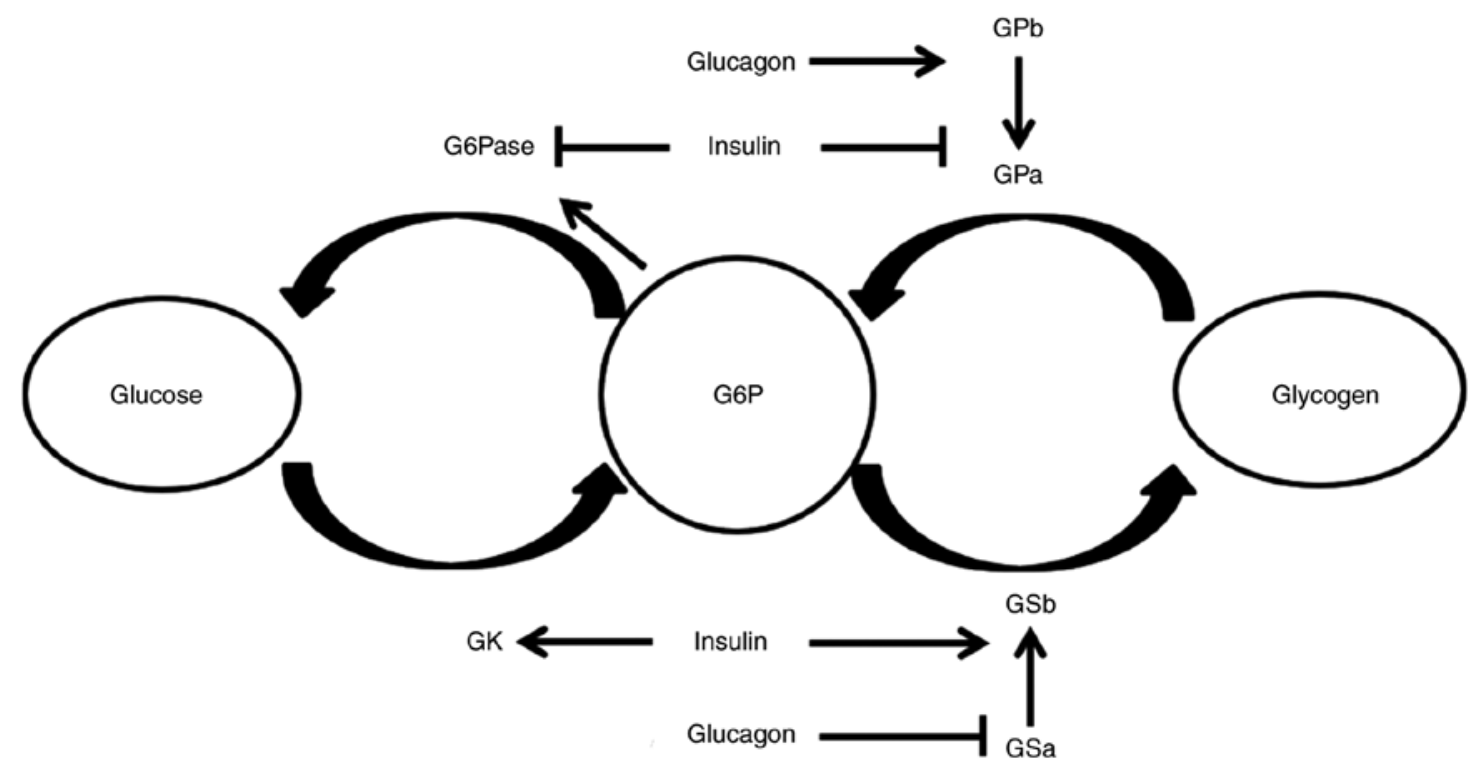

Figure 1. Metabolite cycle of glucose to glycogen. Under the regulation of insulin and glucagon, the liver aids the maintenance of blood glucose homeostasis. Insulin activates the uptake of glucose and the synthesis of glycogen, while inhibiting glycogenolysis and gluconeogenesis. Glucagon inhibits glycogenesis and promotes glycogenolysis. G6P is a key intermediate enzyme of the pathways and elevated G6P levels increases the activity of G6Pase, facilitating the production of glucose. GPa is the active form of GP and GPb is the inactive form. Conversely, GSb is the active form of GS and GSa is its inactive form. G6P, glucose 6-phosphate; GP, glycogen phosphorylase; GS, glycogen synthase; GK, glucokinase; G6Pase, glucose-6-phosphotase.

glycogenolytic molecules and the stimulation of glycogen synthesis molecules, of which glycogenolysis and glycogen synthesis are regulated by complex mechanisms $(35,36)$. Generally, a useful simplification is that glucose is the main inhibitor of hepatic glycogenolysis and insulin is the main activator of hepatic glycogen synthesis. This has been demonstrated in healthy individuals, using $13 \mathrm{C}$ nuclear magnetic resonance spectroscopy (13C-NMRS) measurements of GS and glycogen phosphorylase flux in the liver (37).

The synthesis of glycogen occurs through a process called glycogenesis, which requires ATP and uridine-5'-triphosphate (23). Glycogen can be synthesized directly from glucose (from glucose to G6P to UDP-glucose to glycogen) or indirectly (from glucose to G6P to pyruvate to G6P to UDP-glucose to glycogen) in the liver (23). These pathways have similar effects on hepatic glycogen synthesis (38). Three regulated enzymes have been identified that exert a high degree of control over glycogen metabolism in the liver: GK (39), GS and GP (40). The changes in GK activity are achieved by regulating GK protein expression or the dissociation of GK from the GK regulatory protein (41). The activities of GS and GP are determined by phosphorylation and dephosphorylation, which serve opposite actions on the activity levels of GS and GP enzymes, leading to a large change in glycogen synthesis. After phosphorylation, GP has activity to decompose glycogen, while after dephosphorylation, GS has activity to promote glycogen synthesis (Fig. 1) (23).

Under T1DM conditions, blood glucose levels are high and exogenous administration of insulin is elevated, which leads to increased hepatic glycogen production and excessive glycogen storage in the liver (42). Hepatic glycogenosis is a relatively benign disease and does not progress easily to fibrosis (43); glycogenosis is the hepatic response to the excess circulating insulin and glucose load in patients with T1DM, whereas non-alcoholic steatohepatitis is the more common diagnosis when elevated insulin and glucose levels occur in adults with T2DM (43). The possible pathogenesis of hepatic glycogenosis is considered to occur following the accumulation of hepatic glycogen in patients with unstable DM as a result of the increased flux of glucose into the hepatocytes (44).

T1DM is associated with abnormal hepatic glycogen metabolism; for example Hwang et al previously demonstrated that the net hepatic glycogen synthesis was impaired in poorly controlled patients with T1DM during mixed meals (45). It is also important to note that glycogen synthesis was significantly reduced in patients with poorly controlled T1DM without elevated insulin levels (46). This occurred during the course of a day following the consumption of three isocaloric mixed meals by lean young T1DM [glycated hemoglobin (HbA1c) $8.8 \pm 0.3 \%$ ] and non-diabetic subjects (HbAlc 5.4 $\pm 0.1 \%$ ) (46).

Moreover, 13C-NMRS and variable infusion dual-tracer methodologies were used to study patients with T2DM and non-diabetic volunteer control subjects, which demonstrated that postprandial glycogen synthesis was decreased in mildly overweight patients with T2DM (47). This was accompanied by the impaired inhibition of hepatic glucose production. Patients with T2DM also exhibited a 35\% reduction of hepatic glycogen content (47). Del Prato et al (48) also reported that glycogen synthesis was reduced in patients with T2DM. These results suggested that the reduced glycogen storage in the liver may lead to postprandial hyperglycemia in patients with T2DM. Furthermore, in mice, a previous study highlighted that T2DM hepatic glycogen was present as large aggregates, which may have aided the inhibition of the interconversion between glucose and glycogen in T2DM. In the same study, it was also found that the size of branched glycogen particles was correlated with the glucose release rate (49).

In patients with DM, hepatic glycogen synthesis is impaired; in T1DM, insufficient insulin is responsible for the reduced glycogen synthesis, whereas T2DM is accompanied 
by reduced hepatic glycogen synthesis due to the diminished insulin signaling induced by lipid accumulation in various organs (50). GP inhibitors could thereby increase glycogen synthesis and have been previously used to treat T2DM $(8,51)$.

Decreased hepatic glycogenolysis in DM. Glycogenolysis is not a simple reversal of glycogenesis but a separate pathway, of which two pathways of glycogen breakdown have been identified. The first glycogen breakdown pathway is the canonical glycogenolysis route, including GP and glycogen debranching enzymes (52) (Fig. 1). The other pathway is associated with autophagy (52). Autophagy-dependent glycogen decomposition produces non-phosphorylated glucose through lysosomal 1,4- $\alpha$-glucosidase (52); however, the mechanism by which hepatocytes sense the decline in blood glucose levels and activate selective glycophagy remains poorly understood (53). Hepatic-specific autophagy serves a role in the regulation of blood glucose levels, while insulin has a dominant role over glucagon in the control of liver autophagy (54).

During insulin resistance, glucose inactivates the stimulated form of GP (GPa) and thereby inhibits glycogenolysis (23). During the period of energy restriction in patients with T2DM, the decrease in fasting blood glucose levels is largely due to decreased glycogenolysis, while the change in gluconeogenesis is not significant (55). A previous study aimed to compare the size distribution, degradation kinetics and branching structure of normal and diabetic liver glycogen; it was observed that T2DM hepatic glycogen existed as large, loosely bound aggregates that were not present in the normal liver, which may have resulted in the inflexibility of glucose and glycogen interconversion in T2DM (49).

Impaired glycogenolysis is also noted in T1DM; hepatic glycogen breakdown was significantly reduced in patients with poorly controlled T1DM, as measured by 13C-MNRS (46). In another study, the contribution of net hepatic glycogenolysis during insulin-induced hypoglycemia was quantitatively analyzed using 13C-MNRS in 10 non-diabetic and 7 T1DM subjects $(\mathrm{HbA} 1 \mathrm{c} 6.5 \pm 0.2 \%)$ and it was concluded that in intensively treated T1DM subjects, hypoglycemia failed to stimulate hepatic glycogen decomposition or activate endogenous glucose production (56).

Increased rates of hepatic gluconeogenesis in both TIDM and T2DM subjects. Gluconeogenesis, is essentially a reverse process of the glycolytic pathway. In humans, gluconeogenesis occurs predominantly in the liver (57) and is regulated by a slower mechanism through changes in gene expression (23) in response to hormones, notably insulin and glucagon (36).

Increased glucose production is a consistent feature of T2DM, which can be attributed to increased gluconeogenesis rather than glycogenolysis rates $(58,59)$. The possible influencing factors include: i) Hepatic resistance to the action of insulin, leading to the inappropriate inhibition of hepatic glucose output (60); ii) high levels of glucagon, leading to the overactivation of signaling pathways, which are usually activated during fasting when glucose supply is required (60); and iii) the indirect regulation of gluconeogenesis by excess circulating free fatty acids (FFAs) through the insulin receptor independent pathway $(61,62)$. A previous study using normal blood glucose and hyperglycemic clamps provided evidence for deficiencies in the rapid inhibition of hepatic glucose production in T2DM (63). Non-invasive 13C-NMRS studies have demonstrated that hepatic glycogen decomposition was low and gluconeogenesis was increased in patients with T2DM $(58,64)$. In one study, specific small molecules (SR-18292) were used to increase the acetylation of the peroxisome proliferator-activated receptor gamma coactivator 1-alpha (PGC-1 $\alpha)$. The study demonstrated that these molecules inhibited the activity of PGC- $1 \alpha$-dependent gluconeogenesis, thereby increasing insulin sensitivity and lowering blood glucose levels in T2D mice (65).

T1DM rats were discovered to have $\sim 90 \%$ lower insulin and leptin levels, as well as $90 \%$ higher glucagon levels in the plasma compared with control non-diabetic rats (66). Glucagon stimulates hepatic glucose production by activating enzymes involved in gluconeogenesis and glycogenolysis (60). In animal experiments, the inhibition of glucagon action prevented the metabolic disorders noted in T1DM mice (67). Another study indicated that moderately controlled patients with T1DM exhibited increased glucose production during both rest and exercise, which may account for the increased gluconeogenesis rates (68).

Increased glucose production in the liver that occurs due to enhanced gluconeogenesis is the major contributor to the high blood glucose levels observed in DM (69). Thus, inhibiting gluconeogenesis may provide the most direct route for decreasing glucose production in the liver; for example, Metformin is the most commonly used therapy for T2DM, since it decreases gluconeogenesis in the liver without increasing insulin secretion (70). However, the underlying mechanism by which metformin inhibits hepatic gluconeogenesis remains unknown. The addition of metformin to insulin therapy in T1DM is still under debate and previous findings of metformin in patients with T1DM have resulted in conflicting results (71).

Hepatic glycolytic activity is weakened in DM. Glycolysis is mainly regulated through glucose, insulin and glucagon (9). In addition to increasing hepatic gluconeogenic activity, the inhibition of hepatic glycolysis was found to contribute to elevated blood glucose levels; Henly et al (32) demonstrated that the glycolytic flux in diabetic rat hepatocytes was only $40 \%$ of that noted in normal rat hepatocytes, with a larger proportion of G6P being reconverted to glucose without glycolysis. In a human study, including 7 patients with non-insulin-dependent DM, Del Prato et al (48) demonstrated that glucose uptake was reduced by $54 \%$, with both glycolysis and glucose oxidation being reduced in patients compared with control subjects. In an animal study, STZ-treated mice exhibited significant decreases in GK, phosphofructokinase (PFK1) and pyruvate kinase activity compared with those of control subjects (72).

The pyruvate produced by glycolysis is an important intermediate product in the conversion of carbohydrates to fatty acids and cholesterol (73). The hepatic abnormal regulation of pyruvate metabolism has been reported in DM and NAFLD (74). In insulin-resistant conditions, pyruvate is used for gluconeogenesis and fatty acid synthesis rather than ATP generation, which is promoted by the tricarboxylic acid (TCA) cycle, resulting in hyperglycemia and hepatic steatosis $(75,76)$. The TCA cycle is affected by DM and the glycolytic activity 
is weakened during the development of DM (77). The activation of the pyruvate dehydrogenase complex promotes the conversion of pyruvate to acetyl-CoA by oxidative decarboxylation (78). Go et al (76) reported that by inhibiting pyruvate dehydrogenase kinase 2 , the activity of the hepatic pyruvate dehydrogenase complex was increased, ameliorating hepatic steatosis and improving insulin sensitivity through regulating the TCA cycle.

Glyceraldehyde 3-phosphate dehydrogenase (GAPDH) is an important enzyme used to catalyze the sixth step of glycolysis, which is the step between the energy-requiring phase and the energy-releasing phase (79). GAPDH is a major target protein involved in oxidative stress (80) and it is inactivated due to the accumulation of reactive oxygen species (81). It is well known that oxidative stress serves an important role in the incidence of diabetic complications and hyperglycemia (82). Moreover, it leads to the excessive production of mitochondrial superoxide, resulting in a $66 \%$ decrease in GAPDH activity (83). The imbalance of the nicotinamide adenine dinucleotide (NADH)/NAD ${ }^{+}$redox status in $\mathrm{DM}$ was revealed to inhibit both GAPDH and dihydrolipoamide dehydrogenase in the pyruvate dehydrogenase complex (84) and the impairment of glycolysis led to the accumulation of glyceraldehyde 3-phosphate (G3P), as reported in microvascular and cardiovascular studies (85-87). Therefore, all intermediate products prior to G3P (including G3P) must be converted through the branch pathways of the glycolytic pathway $(82,87,88)$. Moreover, in a previous study GAPDH inactivation resulted in a shift in metabolic flux from glycolysis to the pentose phosphate pathway (PPP) (89); however, to the best of our knowledge, no direct evidence of decreased hepatic GAPDH activity in DM has been previously reported.

In brief, hepatic glycolytic activity is weakened in both T1DM and T2DM, which can enhance hepatic glucose utilization for targeting hepatic glucose output. The alteration of mitochondrial uncoupling can also enhance glucose utilization in the liver by increasing fatty acid and glucose oxidation (90).

Role of the PPP in the diabetic liver is not clear. In the majority of organs, 80-90\% of glucose oxidation occurs via glycolysis and the remaining 10-20\% occurs via the PPP (91); the percentage of glucose metabolized by PPP varies from 5 to $30 \%$ in different tissues (92). In lipid- and steroid-synthesizing tissues, such as the liver, lactating mammary glands, white adipose tissue, adrenal glands and gonads, in addition to the erythrocytes, the PPP produces the highest proportion of glucose flux (92). PPP is usually divided into an oxidative and a nonoxidative pathway and the activity of the former is generally higher than that of the latter (93). G6P dehydrogenase (G6PD) is a rate limiting enzyme of the PPP and produces the majority of nicotinamide adenine dinucleotide phosphate (NADPH) in the cell, which is the most abundant reducing coenzyme in the cells (94). NADPH is an important cofactor involved in several enzymatic reactions in the cell, such as nitric oxide production, fatty acid synthesis/oxidation and the production of glutathione by glutathione reductase (95). In addition, NADPH is required to remove excess hydrogen peroxide from the glutathione system (94).

To the best of our knowledge, the fate of the glucose molecules that are metabolized by the PPP in the liver has not been well studied. In DM, previous findings have demonstrated that the activity of G6PD is inhibited and the content of NADPH is reduced in the liver of T1DM rats (induced by STZ- and alloxan-treatment) (96). Similar findings have also been reported in the liver of patients with chronic DM (97). In an animal study of mild, moderate and severe hyperglycemia induced by STZ and nicotinamide treatment, it was found that the levels of hepatocyte glucose-6-phosphate dehydrogenase (G6PD) activity in mild hyperglycemia remained similar to the normal values, whereas in moderate and severely hyperglycemia, they were significantly reduced (94). The G6PD:NADPH/nicotine adenine dinucleotide phosphate $\left(\mathrm{NADP}^{+}\right)$ratio and the glutathione levels exhibited a negative correlation with the blood glucose concentration and a positive direct correlation with insulin levels (94). In addition, in T2DM obese Zucker rats, hepatic G6PD protein levels and activity were significantly higher compared with those noted in lean rats $(98,99)$. Increased G6PD levels have also been noted in high-fat diet STZ-treated-T2D rats (100).

Hepatic lipogenesis in NAFLD is prevalent in T2DM but not in TIDM. The liver is the primary site of lipogenesis (101) and increased lipogenesis is the major abnormality found in NAFLD (102), while the contribution of dietary fat and blood free fatty acid production are not considerably altered (103). NAFLD is a broad term that includes net triglyceride (TG) deposition in hepatocytes caused by any factor other than ethanol intake and is defined as the presence of more than and/or equal to $5-10 \%$ of fat deposits in hepatocytes (104). The biopsy features in NAFLD include enhanced TG vacuoles, hepatocyte ballooning and necrosis, mixed inflammatory cell infiltration and fibrosis (105).

NAFLD is highly prevalent in T2DM and can also occur in T1DM (106). In children and adolescents with T1DM, NAFLD did not exhibit a significant increase in NAFLD prevalence, as determined by ultrasound screening (107); however, ultrasonography is not the best method to measure fatty liver changes (108). MRI is an accurate imaging technique used for the detection of fatty liver (109). A previous study using MRI with 128 patients with T1DM, 264 patients with T2DM and 67 participants without DM indicated that T1DM was not associated with the increase in the incidence of steatosis (110). Previous studies that used MRI to measure hepatic fat content indicated that the prevalence of NAFLD in adults $(111,112)$ and children patients with T1DM (113) did not increase. In a time-course study of OVE26 mice (a type 1 diabetic model) Oil Red $\mathrm{O}$ staining and biochemical methods were used to detect the lipid content in the liver; the data indicated that uncontrolled T1DM did not cause lipid deposition in the liver, which was most likely due to the reduced lipid synthesis as a result of insulin deficiency (114). NAFLD must be distinguished from the more common glycogen hepatopathy, which is responsible for hepatomegaly and liver dysfunction in patients with T1DM (115). In a study by Cusi et al (112), the prevalence of NAFLD (hepatic fat content $\geq 6 \%$ ) was low in T1DM $(8.8 \%)$ and high in T2DM and a higher prevalence was noted in insulin-naive (75.6\%) versus insulin-treated $(61.7 \%)$ patients with T2DM.

Hepatic metabolic changes in patients with T2DM are characterized via increased liver fat content, impaired insulin clearance and hepatic insulin resistance $(3,116)$. Insulin promotes hepatic lipogenesis and in insulin resistance states, 

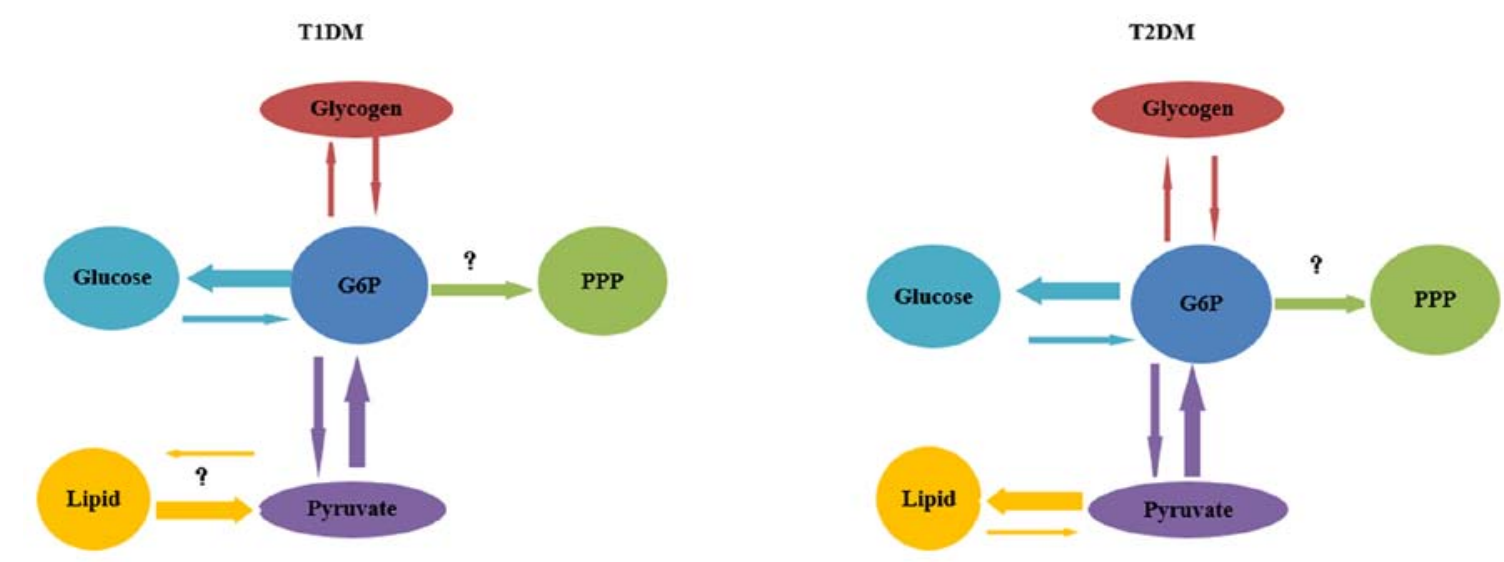

Figure 2. Changes in hepatic glucose metabolic flux in DM. Thick arrows indicate increased flux, whereas thin arrows indicate reduced flux. T1DM: In T1DM, due to the lack of insulin, glycogen synthesis and glycolysis are decreased. The loss of insulin also causes the inactivation of the paracrine regulation of glucagon, which contributes to the development of hyperglucagonemia and to an increase in gluconeogenesis, which in turn leads to hyperglycemia. The changes in hepatic lipolysis in T1DM are not clear. T2DM: Insulin resistance exists in T2DM, leading to decreased glycogen synthesis and glucose metabolism via the TCA cycle, thereby increasing lipogenesis. Moreover, gluconeogenesis flux increases as a result of hyperglucagonemia. The changes in the PPP in both T1DM and T2DM are still not clear. T1DM, type 1 diabetes mellitus; T2DM, type 2 diabetes mellitus; TCA, tricarboxylic acid; PPP, pentose phosphate pathway; G6P, glucose 6-phosphate; ?, unclear.

this action is maintained, whereas its ability to reduce hepatic gluconeogenesis is reported to be impaired $(103,117)$. The production of glucose from non-carbohydrate sources was increased in patients with NAFLD, a phenomenon known as selective insulin resistance (103). This is one explanation for the occurrence of NAFLD in T2DM, whereas another hypothesis is that NAFLD may develop independently of insulin in the liver. Vatner et al (118) reported that in T2DM, the main source of hepatic lipid synthesis was the esterification of preformed fatty acids, which was mainly dependent on the transport of substrates and was largely independent of hepatic insulin action. These findings demonstrated that plasma was the main source of TG synthesis in T2DM albeit the role of insulin in fatty acid esterification (if any) was not clear (119).

Increased liver fat has been discovered to be associated with decreased insulin clearance and sensitivity in patients with T2DM $(116,120)$, whereas in T1DM, which is characterized by decreased insulin secretion, the lipid synthesis in patients with T1DM was reduced $(11,114,121)$. T1DM is characterized by insufficient insulin secretion and excessive glucagon production (2) and the mechanism of NAFLD in T1DM may be complicated; Regnell and Lernmark (122) proposed three hypotheses to explain the occurrence of hepatic steatosis in patients with T1DM. In the present review, two possible reasons are highlighted: Initially, the establishment of insulin therapy was found to promote lipogenesis (113) and weight gain in patients with T1DM (123) and secondly, the increased presence of hepatic lipids may be associated with hyperglycemia that directly influence insulin and glucagon secretion (122). Abnormal autophagy may also have an important role in aggravating lipid metabolic disorders contributing to steatohepatitis in diabetes; Singh et al (124) demonstrated that the inhibition of autophagy in cultured hepatocytes and in mouse liver tissues increased TG storage in lipid droplets.

In brief, hyperglycemia in both T1DM and T2DM may be explained by increased G/G6P cycling or by increased flux through the gluconeogenesis pathway. Reduced flux of glycogenesis and glycogenolysis may also explain the inefficiency of acute regulation of hypoglycemia in fasting and of hyperglycemia following a meal (Fig. 2).

\section{Conclusion}

In the current review, the findings suggested that the dysregulation of hepatic glucose and lipid metabolism may be the primary factor underlying the pathogenesis of T2DM and T1DM. These two chronic diseases affect the body's ability to regulate glucose and result in different hepatic pathological conditions Overall, it is evident that the liver is crucial for systemic glucose homeostasis and that the disorders of glucose metabolism may contribute to the initiation, progression and exacerbation of DM; however, there are still several unanswered questions and gaps in our knowledge that must be addressed in the future.

\section{Acknowledgements}

Not applicable.

\section{Funding}

The present work was supported in part by grants from the Basic Scientific Research Projects of Wenzhou Municipal Science and Technology Bureau (CN) (grant no. Y20180253 to SZJ), NIEHS (T32-ES011564 to JY) and China-U.S. University of Louisville Paediatric Research Exchange Training Program. Personnel expenses and partial research-related expenses for Dr Saizhi Jiang and Dr Kai Wang were provided by the First Affiliated Hospital of the Wenzhou Medical University through a collaborative research agreement between the University of Louisville and the First Affiliated Hospital of the Wenzhou Medical University, Wenzhou, China.

\section{Availability of data and materials}

Data sharing is not applicable to this article, as no data sets were generated or analyzed during the current study. 


\section{Authors' contributions}

SJ, LC, JLY, KW and YQ conceived and designed the present study. All authors read and approved the final manuscript.

\section{Ethics approval and consent to participate}

Not applicable.

\section{Patient consent for publication}

Not applicable.

\section{Competing interests}

The authors declare that they have no competing interests.

\section{References}

1. Towle HC: Glucose as a regulator of eukaryotic gene transcription. Trends Endocrinol Metab 16: 489-494, 2005.

2. Habegger KM, Heppner KM, Geary N, Bartness TJ, DiMarchi R and Tschop $\mathrm{MH}$ : The metabolic actions of glucagon revisited. Nat Rev Endocrinol 6: 689-697, 2010

3. Bhatt HB and Smith RJ: Fatty liver disease in diabetes mellitus. Hepatobiliary Surg Nutr 4: 101-108, 2015.

4. Sumida Y and Yoneda M: Glycogen hepatopathy: An under-recognized hepatic complication of uncontrolled type 1 diabetes mellitus. Intern Med 57: 1063-1064, 2018.

5. Moore MC, Coate KC, Winnick JJ, An Z and Cherrington AD: Regulation of hepatic glucose uptake and storage in vivo. Adv Nutr 3: 286-294, 2012.

6. Petersen MC, Vatner DF and Shulman GI: Regulation of hepatic glucose metabolism in health and disease. Nat Rev Endocrinol 13 572-587, 2017.

7. Dorcely B, Katz K, Jagannathan R, Chiang SS, Oluwadare B, Goldberg IJ and Bergman M: Novel biomarkers for prediabetes, diabetes, and associated complications. Diabetes Metab Syndr Obes 10: 345-361, 2017.

8. Rines AK, Sharabi K, Tavares CD and Puigserver P: Targeting hepatic glucose metabolism in the treatment of type 2 diabetes Nat Rev Drug Discov 15: 786-804, 2016.

9. Rui L: Energy metabolism in the liver. Compr Physiol 4: 177-197, 2014.

10. Girard J: Glucagon, a key factor in the pathophysiology of type 2 diabetes. Biochimie 143: 33-36, 2017.

11. Mittendorfer B and Klein S: Absence of leptin triggers type 1 diabetes. Nat Med 20: 705-706, 2014.

12. Unger RH and Orci L: Paracrinology of islets and the paracrinopathy of diabetes. Proc Natl Acad Sci USA 107: 16009-16012, 2010.

13. Wewer Albrechtsen NJ, Pedersen J, Galsgaard KD, Winther-Sørensen M, Suppli MP, Janah L, Gromada J, Vilstrup H, Knop FK and Holst JJ: The liver- $\alpha$ cell axis and type 2 diabetes. Endocr Rev 40: 1353-1366, 2019.

14. Bergman RN and Iyer MS: Indirect Regulation of endogenous glucose production by insulin: The single gateway hypothesis revisited. Diabetes 66: 1742-1747, 2017.

15. Pearson MJ, Unger RH and Holland WL: Clinical trials, triumphs, and tribulations of glucagon receptor antagonists. Diabetes Care 39: 1075-1077, 2016.

16. Basco D, Zhang Q, Salehi A, Tarasov A, Dolci W, Herrera P, Spiliotis I, Berney X, Tarussio D, Rorsman P and Thorens B: $\alpha$-cell glucokinase suppresses glucose-regulated glucagon secretion. Nat Commun 9: 546, 2018.

17. Quesada I, Tuduri E, Ripoll C and Nadal A: Physiology of the pancreatic alpha-cell and glucagon secretion: Role in glucose homeostasis and diabetes. J Endocrinol 199: 5-19, 2008.

18. Liu S, Ammirati MJ, Song X, Knafels JD, Zhang J, Greasley SE, Pfefferkorn JA and Qiu X: Insights into mechanism of glucokinase activation: Observation of multiple distinct protein conformations. J Biol Chem 287: 13598-13610, 2012.
19. Giordano S, Martocchia A, Toussan L, Stefanelli M, Pastore F, Devito A, Risicato MG, Ruco L and Falaschi P: Diagnosis of hepatic glycogenosis in poorly controlled type 1 diabetes mellitus. World J Diabetes 5: 882-888, 2014.

20. Barzilai N and Rossetti L: Role of glucokinase and glucose-6-phosphatase in the acute and chronic regulation of hepatic glucose fluxes by insulin. J Biol Chem 268: 25019-25025, 1993.

21. Holste LC, Connolly CC, Moore MC, Neal DW and Cherrington AD: Physiological changes in circulating glucagon alter hepatic glucose disposition during portal glucose delivery. Am J Physiol 273: E488-E496, 1997.

22. Ramnanan CJ, Edgerton DS, Kraft G and Cherrington AD: Physiologic action of glucagon on liver glucose metabolism. Diabetes Obes Metab 13 (Suppl 1): S118-S125, 2011.

23. Agius L: Glucokinase and molecular aspects of liver glycogen metabolism. Biochem J 414: 1-18, 2008.

24. Iozzo P, Hallsten K, Oikonen V, Virtanen KA, Kemppainen J, Solin O, Ferrannini E, Knuuti J and Nuutila P: Insulin-mediated hepatic glucose uptake is impaired in type 2 diabetes: Evidence for a relationship with glycemic control. J Clin Endocrinol Metab 88: 2055-2060, 2003.

25. Coate KC, Kraft G, Shiota M, Smith MS, Farmer B, Neal DW, Williams P, Cherrington AD and Moore MC: Chronic overeating impairs hepatic glucose uptake and disposition. Am J Physiol Endocrinol Metab 308: E860-E867, 2015.

26. Watanabe H, Inaba Y, Kimura K, Matsumoto M, Kaneko S, Kasuga $M$ and Inoue H: Sirt2 facilitates hepatic glucose uptake by deacetylating glucokinase regulatory protein. Nat Commun 9: 30, 2018.

27. van Dijk TH, van der Sluijs FH, Wiegman $\mathrm{CH}$, Baller JF, Gustafson LA, Burger HJ, Herling AW, Kuipers F, Meijer AJ and Reijngoud DJ: Acute inhibition of hepatic glucose-6-phosphatase does not affect gluconeogenesis but directs gluconeogenic flux toward glycogen in fasted rats. A pharmacological study with the chlorogenic acid derivative S4048. J Biol Chem 276 25727-25735, 2001.

28. Foufelle F and Ferré P: New perspectives in the regulation of hepatic glycolytic and lipogenic genes by insulin and glucose: A role for the transcription factor sterol regulatory element binding protein-1c. Biochem J 366: 377-391, 2002.

29. Clore JN, Stillman J and Sugerman H: Glucose-6-phosphatase flux in vitro is increased in type 2 diabetes. Diabetes 49: 969-974, 2000.

30. Bandsma RH, Grefhorst A, van Dijk TH, van der Sluijs FH, Hammer A, Reijngoud DJ and Kuipers F: Enhanced glucose cycling and suppressed de novo synthesis of glucose-6-phosphate result in a net unchanged hepatic glucose output in ob/ob mice. Diabetologia 47: 2022-2031, 2004.

31. Rooney DP, Neely RD, Beatty O, Bell NP, Sheridan B, Atkinson AB, Trimble ER and Bell PM: Contribution of glucose/glucose 6-phosphate cycle activity to insulin resistance in type 2 (non-insulin-dependent) diabetes mellitus. Diabetologia 36: 106-112, 1993.

32. Henly DC, Phillips JW and Berry MN: Suppression of glycolysis is associated with an increase in glucose cycling in hepatocytes from diabetic rats. J Biol Chem 271: 11268-11271, 1996.

33. Torres TP, Catlin RL, Chan R, Fujimoto Y, Sasaki N, Printz RL, Newgard CB and Shiota M: Restoration of hepatic glucokinase expression corrects hepatic glucose flux and normalizes plasma glucose in zucker diabetic fatty rats. Diabetes 58: 78-86, 2009.

34. Vella A, Freeman JLR, Dunn I, Keller K, Buse JB and Valcarce C: Targeting hepatic glucokinase to treat diabetes with TTP399, a hepatoselective glucokinase activator. Sci Transl Med 11: eaau3441, 2019.

35. Ferrer JC, Favre C, Gomis RR, Fernández-Novell JM, García-Rocha M, de la Iglesia N, Cid E and Guinovart JJ: Control of glycogen deposition. FEBS Lett 546: 127-132, 2003.

36. Lin HV and Accili D: Hormonal regulation of hepatic glucose production in health and disease. Cell Metab 14: 9-19, 2011.

37. Petersen KF, Laurent D, Rothman DL, Cline GW and Shulman GI: Mechanism by which glucose and insulin inhibit net hepatic glycogenolysis in humans. J Clin Invest 101: 1203-1209, 1998.

38. Soares AF, Viega FJ, Carvalho RA and Jones JG: Quantifying hepatic glycogen synthesis by direct and indirect pathways in rats under normal ad libitum feeding conditions. Magn Reson Med 61: 1-5, 2009

39. Agius L, Peak M, Newgard CB, Gomez-Foix AM and Guinovart JJ: Evidence for a role of glucose-induced translocation of glucokinase in the control of hepatic glycogen synthesis. J Biol Chem 271: 30479-30486, 1996. 
40. Aiston S, Hampson L, Gómez-Foix AM, Guinovart JJ and Agius L: Hepatic glycogen synthesis is highly sensitive to phosphorylase activity: Evidence from metabolic control analysis. J Biol Chem 276: 23858-23866, 2001.

41. Matschinsky FM and Magnuson MA (eds): Glucokinase and Glycemic Diseases: From Basics to Novel Therapeutics. Karger, Basel, pp1-9, 2004.

42. Satyarengga M, Zubatov Y, Frances S, Narayanswami G and Galindo RJ: Glycogenic hepatopathy: A complication of uncontrolled diabetes. AACE Clin Case Rep 3: e255-e259, 2017.

43. Chatila $\mathrm{R}$ and West AB: Hepatomegaly and abnormal liver tests due to glycogenosis in adults with diabetes. Medicine (Baltimore) 75: 327-333, 1996.

44. Julián MT, Alonso N, Ojanguren I, Pizarro E, Ballestar E and Puig-Domingo M: Hepatic glycogenosis: An underdiagnosed complication of diabetes mellitus? World J Diabetes 6: 321-325, 2015

45. Hwang JH, Perseghin G, Rothman DL, Cline GW, Magnusson I, Petersen KF and Shulman GI: Impaired net hepatic glycogen synthesis in insulin-dependent diabetic subjects during mixed meal ingestion. A 13C nuclear magnetic resonance spectroscopy study. J Clin Invest 95: 783-787, 1995.

46. Bischof MG, Krssak M, Krebs M, Bernroider E, Stingl H, Waldhäusl W and Roden M: Effects of short-term improvement of insulin treatment and glycemia on hepatic glycogen metabolism in type 1 diabetes. Diabetes 50: 392-398, 2001

47. Krssak M, Brehm A, Bernroider E, Anderwald C, Nowotny P, Dalla Man C, Cobelli C, Cline GW, Shulman GI, Waldhäusl W and Roden M: Alterations in postprandial hepatic glycogen metabolism in type 2 diabetes. Diabetes 53: 3048-3056, 2004.

48. Del Prato S, Bonadonna RC, Bonora E, Gulli G, Solini A, Shank M and DeFronzo RA: Characterization of cellular defects of insulin action in type 2 (non-insulin-dependent) diabetes mellitus. J Clin Invest 91: 484-494, 1993.

49. Besford QA,Zeng XY, Ye JM and Gray-Weale A: Liver glycogen in type 2 diabetic mice is randomly branched as enlarged aggregates with blunted glucose release. Glycoconj J 33: 41-51, 2016.

50. Samuel VT and Shulman GI: The pathogenesis of insulin resistance: Integrating signaling pathways and substrate flux. J Clin Invest 126: 12-22, 2016

51. Henke BR and Sparks SM: Glycogen phosphorylase inhibitors. Mini Rev Med Chem 6: 845-857, 2006.

52. Ha J, Guan KL and Kim J: AMPK and autophagy in glucose/glycogen metabolism. Mol Aspects Med 46: 46-62, 2015.

53. Madrigal-Matute J and Cuervo AM: Regulation of liver metabolism by autophagy. Gastroenterology 150: 328-339, 2016.

54. Ezaki J,Matsumoto N, Takeda-Ezaki M, Komatsu M, Takahashi K, Hiraoka Y, Taka H, Fujimura T, Takehana K, Yoshida M, et al Liver autophagy contributes to the maintenance of blood glucose and amino acid levels. Autophagy 7: 727-736, 2011

55. Christiansen MP, Linfoot PA, Neese RA and Hellerstein MK: Effect of dietary energy restriction on glucose production and substrate utilization in type 2 diabetes. Diabetes 49: 1691-1699, 2000.

56. Kishore P, Gabriely I, Cui MH, Di Vito J, Gajavelli S, Hwang JH and Shamoon H: Role of hepatic glycogen breakdown in defective counterregulation of hypoglycemia in intensively treated type 1 diabetes. Diabetes 55: 659-666, 2006.

57. Ekberg K, Landau BR, Wajngot A, Chandramouli V, Efendic S, Brunengraber $\mathrm{H}$ and Wahren J: Contributions by kidney and liver to glucose production in the postabsorptive state and after $60 \mathrm{~h}$ of fasting. Diabetes 48: 292-298, 1999.

58. Hundal RS, Krssak M, Dufour S, Laurent D, Lebon V, Chandramouli V, Inzucchi SE, Schumann WC, Petersen KF, Landau BR and Shulman GI: Mechanism by which metformin reduces glucose production in type 2 diabetes. Diabetes 49: 2063-2069, 2000.

59. Rizza RA: Pathogenesis of fasting and postprandial hyperglycemia in type 2 diabetes: Implications for therapy. Diabetes 59: 2697-2707, 2010.

60. Sharabi K, Tavares CD, Rines AK and Puigserver P: Molecular pathophysiology of hepatic glucose production. Mol Aspects Med 46: 21-33, 2015.

61. Kehlenbrink S, Koppaka S, Martin M, Relwani R, Cui MH, Hwang JH, Li Y, Basu R, Hawkins M and Kishore P: Elevated NEFA levels impair glucose effectiveness by increasing net hepatic glycogenolysis. Diabetologia 55: 3021-3028, 2012.

62. Titchenell PM, Quinn WJ, Lu M, Chu Q, Lu W, Li C, Chen H, Monks BR, Chen J, Rabinowitz JD and Birnbaum MJ: Direct Hepatocyte insulin signaling is required for lipogenesis but is dispensable for the suppression of glucose production. Cell Metab 23: 1154-1166, 2016.
63. Hawkins M, Gabriely I, Wozniak R, Reddy K, Rossetti L and Shamoon H: Glycemic control determines hepatic and peripheral glucose effectiveness in type 2 diabetic subjects. Diabetes 51: 2179-2189, 2002.

64. Magnusson I, Rothman DL, Katz LD, Shulman RG and Shulman GI: Increased rate of gluconeogenesis in type II diabetes mellitus. A $13 \mathrm{C}$ nuclear magnetic resonance study. J Clin Invest 90: 1323-1327, 1992.

65. Sharabi K, Lin H, Tavares CDJ, Dominy JE, Camporez JP, Perry RJ, Schilling R, Rines AK, Lee J, Hickey M, et al: Selective chemical inhibition of PGC-1 $\alpha$ gluconeogenic activity ameliorates type 2 diabetes. Cell 169: 148-160.e15, 2017.

66. Perry RJ, Zhang XM, Zhang D, Kumashiro N, Camporez JP, Cline GW, Rothman DL and Shulman GI: Leptin reverses diabetes by suppression of the hypothalamic-pituitary-adrenal axis. Nat Med 20: 759-763, 2014.

67. Lee Y, Wang MY, Du XQ, Charron MJ and Unger RH: Glucagon receptor knockout prevents insulin-deficient type 1 diabetes in mice. Diabetes 60: 391-397, 2011.

68. Petersen KF, Price TB and Bergeron R: Regulation of net hepatic glycogenolysis and gluconeogenesis during exercise: Impact of type 1 diabetes. J Clin Endocrinol Metab 89: 4656-4664, 2004.

69. Hatting M, Tavares CDJ, Sharabi K, Rines AK and Puigserver P: Insulin regulation of gluconeogenesis. Ann NY Acad Sci 1411: 21-35, 2018

70. Madiraju AK, Erion DM, Rahimi Y, Zhang XM, Braddock DT, Albright RA, Prigaro BJ, Wood JL, Bhanot S, MacDonald MJ, et al: Metformin suppresses gluconeogenesis by inhibiting mitochondrial glycerophosphate dehydrogenase. Nature 510: 542-546, 2014.

71. Sabet S, Condren ME, Boston AF, Doak LC and Chalmers LJ: Evolving pharmacotherapeutic strategies for type 1 diabetes mellitus. J Pediatr Pharmacol Ther 23: 351-361, 2018.

72. Abdulrazaq NB, Cho MM, Win NN, Zaman R and Rahman MT: Beneficial effects of ginger (Zingiber officinale) on carbohydrate metabolism in streptozotocin-induced diabetic rats. Br J Nutr 108: 1194-1201, 2012.

73. Gray LR, Tompkins SC and Taylor EB: Regulation of pyruvate metabolism and human disease. Cell Mol Life Sci 71: 2577-2604, 2014.

74. Cotter DG, Ercal B, Huang X, Leid JM, d'Avignon DA, Graham MJ, Dietzen DJ, Brunt EM, Patti GJ and Crawford PA: Ketogenesis prevents diet-induced fatty liver injury and hyperglycemia. J Clin Invest 124: 5175-5190, 2014.

75. Kumashiro N, Beddow SA, Vatner DF, Majumdar SK, Cantley JL, Guebre-Egziabher F, Fat I, Guigni B, Jurczak MJ, Birkenfeld AL, et al: Targeting pyruvate carboxylase reduces gluconeogenesis and adiposity and improves insulin resistance. Diabetes 62: 2183-2194, 2013.

76. Go Y, Jeong JY, Jeoung NH, Jeon JH, Park BY, Kang HJ, Ha CM, Choi YK, Lee SJ, Ham HJ, et al: Inhibition of pyruvate dehydrogenase kinase 2 protects against hepatic steatosis through modulation of tricarboxylic acid cycle anaplerosis and ketogenesis. Diabetes 65: 2876-2887, 2016.

77. Chen M, Zheng H, Xu M, Zhao L, Zhang Q, Song J, Zhao Z, $\mathrm{Lu} \mathrm{S}$, Weng Q, Wu X, et al: Changes in hepatic metabolic profile during the evolution of STZ-induced diabetic rats via an ${ }^{1} \mathrm{H}$ NMR-based metabonomic investigation. Biosci Rep: Apr 23, 2019 (Epub ahead of print). doi: 10.1042/BSR20181379.

78. Sugden MC and Holness MJ: Recent advances in mechanisms regulating glucose oxidation at the level of the pyruvate dehydrogenase complex by PDKs. Am J Physiol Endocrinol Metab 284: E855-E862, 2003

79. Seidler NW: GAPDH and intermediary metabolism. Adv Exp Med Biol 985: 37-59, 2013.

80. Hwang NR, Yim SH, Kim YM, Jeong J, Song EJ, Lee Y, Lee JH, Choi S and Lee KJ: Oxidative modifications of glyceraldehyde-3-phosphate dehydrogenase play a key role in its multiple cellular functions. Biochem J 423: 253-264, 2009.

81. Yun J, Mullarky E, Lu C, Bosch KN, Kavalier A, Rivera K, Roper J, Chio II, Giannopoulou EG, Rago C, et al: Vitamin C selectively kills KRAS and BRAF mutant colorectal cancer cells by targeting GAPDH. Science 350: 1391-1396, 2015.

82. Giacco F and Brownlee M: Oxidative stress and diabetic complications. Circ Res 107: 1058-1070, 2010.

83. Du XL, Edelstein D, Rossetti L, Fantus IG, Goldberg H, Ziyadeh F, Wu J and Brownlee M: Hyperglycemia-induced mitochondrial superoxide overproduction activates the hexosamine pathway and induces plasminogen activator inhibitor-1 expression by increasing Sp1 glycosylation. Proc Natl Acad Sci USA 97: 12222-12226, 2000 
84. Wu J, Jin Z, Zheng $\mathrm{H}$ and Yan LJ: Sources and implications of $\mathrm{NADH} / \mathrm{NAD}(+)$ redox imbalance in diabetes and its complications. Diabetes Metab Syndr Obes 9: 145-153, 2016.

85. Funk SD, Yurdagul A Jr and Orr AW: Hyperglycemia and endothelial dysfunction in atherosclerosis: Lessons from type 1 diabetes. Int J Vasc Med 2012: 569654, 2012.

86. Rask-Madsen C and King GL: Vascular complications of diabetes: Mechanisms of injury and protective factors. Cell Metab 17: 20-33, 2013

87. Yan LJ: Pathogenesis of chronic hyperglycemia: From reductive stress to oxidative stress. J Diabetes Res 2014: 137919, 2014

88. Brownlee M: The pathobiology of diabetic complications: A unifying mechanism. Diabetes 54: 1615-1625, 2005.

89. Ralser M, Wamelink MM, Kowald A, Gerisch B, Heeren G, Struys EA, Klipp E, Jakobs C, Breitenbach M, Lehrach H and Krobitsch S: Dynamic rerouting of the carbohydrate flux is key to counteracting oxidative stress. J Biol 6: 10, 2007.

90. Perry RJ, Kim T, Zhang XM, Lee HY, Pesta D, Popov VB, Zhang D, Rahimi Y, Jurczak MJ, Cline GW, et al: Reversal of hypertriglyceridemia, fatty liver disease, and insulin resistance by a liver-targeted mitochondrial uncoupler. Cell Metab 18: 740-748, 2013.

91. Wamelink MM, Struys EA and Jakobs C: The biochemistry, metabolism and inherited defects of the pentose phosphate pathway: A review. J Inherit Metab Dis 31: 703-717, 2008.

92. Riganti C, Gazzano E, Polimeni M, Aldieri E and Ghigo D: The pentose phosphate pathway: An antioxidant defense and a crossroad in tumor cell fate. Free Radic Biol Med 53: 421-436, 2012.

93. Cabezas H, Raposo RR and Meléndez-Hevia E: Activity and metabolic roles of the pentose phosphate cycle in several rat tissues. Mol Cell Biochem 201: 57-63, 1999.

94. Díaz-Flores M, Ibáñez-Hernández MA, Galván RE, Gutiérrez M, Durán-Reyes G, Medina-Navarro R Pascoe-Lira D, Ortega-Camarillo C, Vilar-Rojas C, Cruz M and Baiza-Gutman LA: Glucose-6-phosphate dehydrogenase activity and NADPH/NADP ${ }^{+}$ratio in liver and pancreas are dependent on the severity of hyperglycemia in rat. Life Sci 78: 2601-2607, 2006.

95. Spaans SK, Weusthuis RA, van der Oost J and Kengen SW: NADPH-generating systems in bacteria and archaea. Front Microbiol 6: 742, 2015

96. Aragno M, Tamagno E, Gatto V, Brignardello E, Parola S, Danni $\mathrm{O}$ and Boccuzzi G: Dehydroepiandrosterone protects tissues of streptozotocin-treated rats against oxidative stress Free Radic Biol Med 26: 1467-1474, 1999.

97. Cédola N, Cabarrou A, Auciello N, Doria I, Ponce de León H and Baylon N: The liver in human diabetes. Concentration of some induced enzymes. Acta Diabetol Lat 12: 263-271, 1975.

98. Gupte RS, Floyd BC, Kozicky M, George S, Ungvari ZI, Neito V, Wolin MS and Gupte SA: Synergistic activation of glucose-6-phosphate dehydrogenase and $\mathrm{NAD}(\mathrm{P}) \mathrm{H}$ oxidase by Src kinase elevates superoxide in type 2 diabetic, Zucker fa/fa, rat liver. Free Radic Biol Med 47: 219-228, 2009.

99. Shepherd A and Cleary MP: Metabolic alterations after dehydroepiandrosterone treatment in Zucker rats. Am J Physiol 246: E123-E128, 1984.

100. Dong K, Ni H, Wu M, Tang Z, Halim M and Shi D: ROS-mediated glucose metabolic reprogram induces insulin resistance in type 2 diabetes. Biochem Biophys Res Commun 476: 204-211, 2016.

101. Kolderup A and Svihus B: Fructose metabolism and relation to atherosclerosis, type 2 diabetes, and obesity. J Nutr Metab 2015 $823081,2015$.

102. Lambert JE, Ramos-Roman MA, Browning JD and Parks EJ: Increased de novo lipogenesis is a distinct characteristic of individuals with nonalcoholic fatty liver disease. Gastroenterology 146: 726-735, 2014.

103. Softic S, Cohen DE and Kahn CR: Role of dietary fructose and hepatic de novo lipogenesis in fatty liver disease. Dig Dis Sci 61 1282-1293, 2016.

104. Barros BSV, Santos DC, Pizarro MH, del Melo LGN and Gomes MB: Type 1 diabetes and non-alcoholic fatty liver disease: When should we be concerned? A nationwide study in Brazil. Nutrients 9: E878, 2017.

105. Calzadilla Bertot L and Adams LA: The natural course of non-alcoholic fatty liver disease. Int J Mol Sci 17: E774, 2016.

106. Targher G, Bertolini L, Padovani R, Rodella S, Zoppini G, Pichiri I, Sorgato C, Zenari L and Bonora E: Prevalence of non-alcoholic fatty liver disease and its association with cardiovascular disease in patients with type 1 diabetes. J Hepatol 53: 713-718, 2010.
107. Kummer S, Klee D, Kircheis G, Friedt M, Schaper J, Häussinger D, Mayatepek E and Meissner T: Screening for non-alcoholic fatty liver disease in children and adolescents with type 1 diabetes mellitus: A cross-sectional analysis. Eur J Pediatr 176: 529-536, 2017

108. Lăpădat AM, Jianu IR, Ungureanu BS, Florescu LM, Gheonea DI, Sovaila S and Gheonea IA: Non-invasive imaging techniques in assessing non-alcoholic fatty liver disease: A current status of available methods. J Med Life 10: 19-26, 2017.

109. Guiu B, Petit JM, Loffroy R, Ben Salem D, Aho S, Masson D, Hillon P, Krause D and Cercueil JP, Quantification of liver fat content: Comparison of triple-echo chemical shift gradient-echo imaging and in vivo proton MR spectroscopy. Radiology 250: 95-102, 2009.

110. Petit JM, Pedro L, Guiu B, Duvillard L, Bouillet B, Jooste V, Habchi M, Crevisy E, Fourmont C, Buffier P, et al: Type 1 diabetes is not associated with an increased prevalence of hepatic steatosis. Diabet Med 32: 1648-1651, 2015.

111. Perseghin G, Lattuada G, De Cobelli F, Esposito A, Costantino F, Canu T, Scifo P, De Taddeo F, Maffi P, Secchi A, et al: Reduced intrahepatic fat content is associated with increased whole-body lipid oxidation in patients with type 1 diabetes. Diabetologia 48: 2615-2621, 2005.

112. Cusi K, Sanyal AJ, Zhang S, Hartman ML, Bue-Valleskey JM, Hoogwerf BJ and Haupt A: Non-alcoholic fatty liver disease (NAFLD) prevalence and its metabolic associations in patients with type 1 diabetes and type 2 diabetes. Diabetes Obes Metab 19: 1630-1634, 2017.

113. Regnell SE, Peterson P, Trinh L, Broberg P, Leander P, Lernmark A, Månsson S and Elding Larsson H: Magnetic resonance imaging reveals altered distribution of hepatic fat in children with type 1 diabetes compared to controls. Metabolism 64: 872-878, 2015.

114. Jiang S, Tang X, Wang K, Liang Y, Qian Y, Lu C and Cai L: Hepatic functional and pathological changes of type 1 diabetic mice in growing and maturation time. J Cell Mol Med 23: 5794-5807, 2019 .

115. Torbenson M, Chen YY, Brunt E, Cummings OW, Gottfried M, Jakate S, Liu YC, Yeh MM and Ferrell L: Glycogenic hepatopathy: An underrecognized hepatic complication of diabetes mellitus. Am J Surg Pathol 30: 508-513, 2006.

116. Kotronen A, Juurinen L, Tiikkainen M, Vehkavaara S and Yki-Järvinen H: Increased liver fat, impaired insulin clearance, and hepatic and adipose tissue insulin resistance in type 2 diabetes. Gastroenterology 135: 122-130, 2008.

117. Titchenell PM, Lazar MA and Birnbaum MJ: Unraveling the regulation of hepatic metabolism by insulin. Trends Endocrinol Metab 28: 497-505, 2017.

118. Vatner DF, Majumdar SK, Kumashiro N, Petersen MC, Rahimi Y, Gattu AK, Bears M, Camporez JP, Cline GW, Jurczak MJ, et al: Insulin-independent regulation of hepatic triglyceride synthesis by fatty acids. Proc Natl Acad Sci USA 112: 1143-1148, 2015.

119. Osório J: Diabetes: Hepatic lipogenesis independent of insulin in type 2 diabetes mellitus-a paradox clarified. Nat Rev Endocrinol 11: 130,2015.

120. Alwahsh SM, Dwyer BJ, Forbes S, Thiel DH, Lewis PJ and Ramadori G: Insulin production and resistance in different models of diet-induced obesity and metabolic syndrome. Int J Mol Sci 18: pii: E285, 2017.

121. American Diabetes Association: Diagnosis and classification of diabetes mellitus. Diabetes Care 35 (Suppl 1): S64-S71, 2012.

122. Regnell SE and Lernmark $\AA$ : Hepatic steatosis in type 1 diabetes. Rev Diabet Stud 8: 454-467, 2011.

123. Purnell JQ, Zinman B and Brunzell JD; DCCT/EDIC Research Group: The effect of excess weight gain with intensive diabetes mellitus treatment on cardiovascular disease risk factors and atherosclerosis in type 1 diabetes mellitus: Results from the diabetes control and complications trial/epidemiology of diabetes interventions and complications study (DCCT/EDIC) study. Circulation 127: 180-187, 2013

124. Singh R, Kaushik S, Wang Y, Xiang Y, Novak I, Komatsu M, Tanaka K, Cuervo AM and Czaja MJ: Autophagy regulates lipid metabolism. Nature 458: 1131-1135, 2009.

This work is licensed under a Creative Commons Attribution-NonCommercial-NoDerivatives 4.0 International (CC BY-NC-ND 4.0) License. 